\title{
Erythropoietin signaling promotes oligodendrocyte development following prenatal systemic hypoxic-ischemic brain injury
}

Lauren L. Jantzie', Robert H. Miller ${ }^{2}$ and Shenandoah Robinson ${ }^{1}$

BACKGROUND: Brain injury from preterm birth causes white matter injury (WMI), and it leads to chronic neurological deficits including cerebral palsy, epilepsy, cognitive, and behavioral delay. Immature O4+ oligodendrocytes are particularly vulnerable to WMI. Understanding how the developing brain recovers after injury is essential to finding more effective therapeutic strategies. Erythropoietin (EPO) promotes neuronal recovery after injury; however, its role in enhancing oligodendroglial lineage recovery is unclear. Previously, we found that recombinant EPO (rEPO) treatment enhances myelin basic protein (MBP) expression and functional recovery in adult rats after prenatal transient systemic hypoxia-ischemia (TSHI). We hypothesized that after injury, rEPO would enhance oligodendroglial lineage cell genesis, survival, maturation, and myelination.

METHODS: I $n$ vitro assays were used to define how rEPO contributes to specific stages of oligodendrocyte development and recovery after TSHI.

RESULTS: After prenatal TSHI injury, rEPO promotes genesis of oligodendrocyte progenitors from oligodendrospheres, survival of oligodendrocyte precursor cells (OPCs) and O4+ immature oligodendrocytes, $\mathrm{O} 4+$ cell process extension, and MBP expression. rEPO did not alter OPC proliferation.

CONCLUSION: Together, these studies demonstrate that EPO signaling promotes critical stages of oligodendroglial lineage development and recovery after prenatal TSHI injury. EPO treatment may be beneficial to preterm and other infant patient populations with developmental brain injury hallmarked by WMI.

$\mathbf{P}$ erinatal brain injury (PBI) from preterm birth causes white matter injury (WMI) with loss of oligodendrocytes and axons throughout the central nervous system (1). After commitment to the oligodendrocyte lineage, platelet-derived growth factor- $\alpha$ receptor (PDGFaR)-expressing oligodendrocyte precursor cells (OPCs) proliferate and differentiate into process-extending $\mathrm{O} 4$-immunopositive immature oligodendrocytes, finally becoming mature myelin basic protein (MBP)-forming oligodendrocytes. Each step in oligodendroglial lineage development is precisely regulated by intrinsic and environmental factors to ensure the correct amount of myelin forms in the correct location at the correct time. Waves of OPCs are generated throughout the perinatal period (2). Alterations in the local environment following PBI hinder the maturation of newly generated OPCs required to replenish lost oligodendrocytes (3). Multiple mechanisms contribute to the developmental vulnerability of the oligodendrocyte lineage to injury, including free radical scavenging enzymes, glutamate receptor activation, and inflammation $(1,4)$.

Erythropoietin (EPO) signaling supports neuronal development and enhances neuronal recovery after injury in both developing and mature brain (5). EPO receptors (EPOR) are present in all the stages of oligodendroglial lineage cells $(6,7)$, and EPOR are upregulated on OPCs and immature oligodendrocytes after prenatal injury (6). Signaling through EPOR regulates neural cell survival during the development (8). While ligand binding to EPOR activates intracellular survival pathways, unbound EPOR predispose cells to apoptosis (8-10). Systemic exogenous postnatal recombinant EPO (rEPO) treatment following prenatal transient systemic hypoxia-ischemia (TSHI) attenuates MBP loss in adult rats (6). EPO treatment in other models of PBI has also shown improved MBP expression (11-15). Prenatal injury increases EPOR expression on developing neural cells without an adequate concomitant increase in ligand expression (6). Therefore, we predicted that EPOR+ immature oligodendrocytes were less likely to survive after TSHI in the absence of adequate EPO ligand. In addition, the role of exogenous rEPO treatment on specific developmental processes involved in the generation of mature oligodendrocytes and their recovery after injury remain poorly defined. Therefore, we delineate here how the rEPO treatment impacts oligodendroglial lineage development including OPC generation from stem cells in oligodendrospheres, OPC proliferation, immature oligodendrocyte survival and differentiation, mature oligodendrocyte process extension, and MBP expression using in vitro assays. Furthermore, we compare how the oligodendroglial lineage response to rEPO is altered following prenatal TSHI that mimics brain injury suffered by a subset of human infants born very preterm. We hypothesized that following 
TSHI injury, rEPO enhances the genesis, survival, differentiation, and maturation of oligodendroglial lineage cells.

\section{RESULTS}

\section{EPO Promotes Oligodendrogenesis}

EPO enhances neurogenesis $(16,17)$. To determine whether rEPO also enhances genesis of oligodendrocyte lineage progenitors, oligodendrospheres were generated from embryonic day (E) 19 brains (18). Addition of rEPO increased the size of oligodendrospheres from both sham and TSHI brains (Figure 1a). In the absence of rEPO, most immunolabeled cells migrating from sham oligodendrospheres had a bipolar morphology typical of OPCs. By contrast, in TSHI oligodendrospheres without $\mathrm{rEPO}$, far fewer A2B5+ or O4+ cells were present. On addition of rEPO, oligodendrospheres from both sham and TSHI cultures had more A2B5+/O4+ cells present, and most immunolabeled cells had multiple processes consistent with an $\mathrm{O} 4+$ immature oligodendrocyte morphology.

To quantify induction of OPCs by rEPO, quantitative PCR was performed to measure PDGFaR mRNA, an OPC-specific marker not expressed on other glial or neuronal cells (Figure 1b). In sham oligodendrospheres, $\mathrm{rEPO}(5 \mathrm{U} / \mathrm{ml})$ induced a moderate $(74 \%)$ increase in PDGFaR mRNA levels as compared with
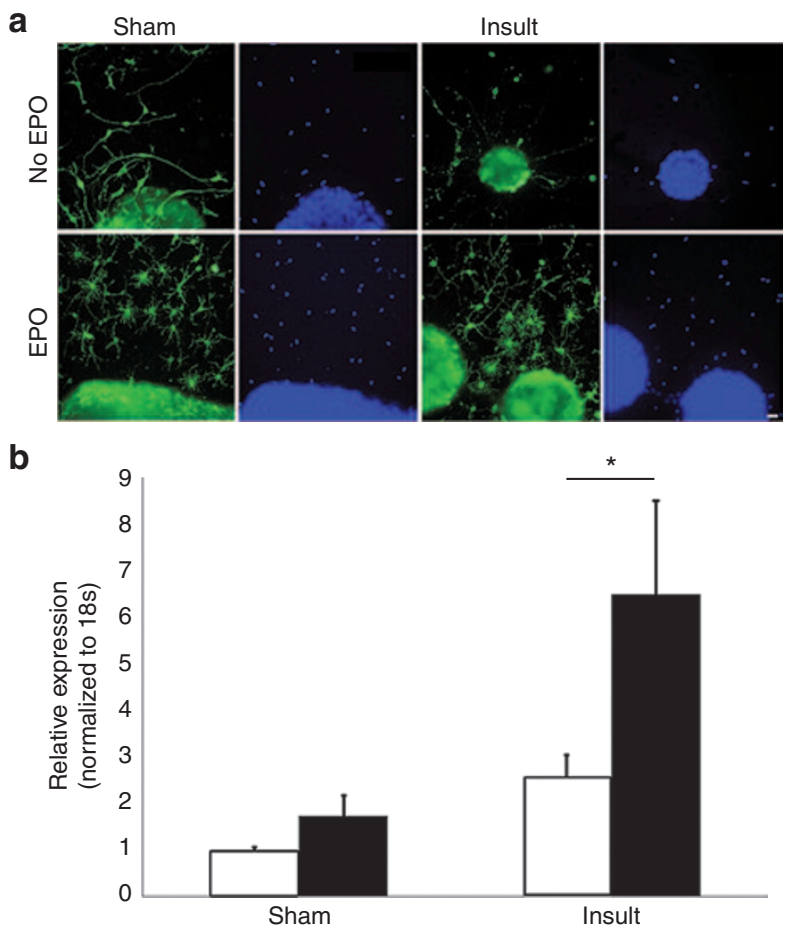

Figure 1. Recombinant erythropoietin (rEPO) promotes oligodendrocyte precursor cell (OPC) genesis in oligodendrospheres. (a) Photomicrograph of oligodendrospheres immunolabeled with $\mathrm{A} 2 \mathrm{~B} 5$ and $\mathrm{O} 4$ antibodies (green). Following prenatal TSHI, fewer immunolabeled cells migrate from oligodendrospheres, and overall oligodendrospheres are smaller. With rEPO treatment, oligodendrospheres are larger, with more immunolabeled cells with multiple processes. DAPI+ nuclei are labeled blue. Bar $=10 \mu \mathrm{m}$. (b) PDGFaR quantitative PCR. Prenatal TSHI induces a 2.7-fold increase in PRGFaR mRNA in vehicle-treated cultures (white columns). rEPO treatment $(5 \mathrm{U} / \mathrm{ml}$, black columns) increased PDGFaR mRNA, particularly in TSHI cultures (two-way ANOVA, ${ }^{*} P=0.044$ ). oligodendrospheres with no rEPO treatment. As compared with shams, prenatal TSHI induced a 2.6-fold increase in PDGFaR transcripts. In TSHI cultures, rEPO treatment significantly increased PDGFaR mRNA $(P=0.044)$. EPOR mRNA levels were $55 \%$ higher in TSHI oligodendrospheres $(0.73 \pm 0.11)$, as compared with those from shams $(0.47 \pm 0.15)$, consistent with an increase in EPOR found on neural cells after PBI (6).

\section{EPO Signaling Enhances Immature Oligodendrocyte Survival After Injury}

Immature oligodendrocytes are particularly vulnerable to cell death following brain injury from preterm birth $(19,20)$. To test whether EPO signaling alters immature oligodendrocyte survival after TSHI, postnatal day (P) 2 mixed cell cultures were incubated $3 \mathrm{~d}$ in vitro (DIV). Exposure to $\mathrm{rEPO}(5 \mathrm{U} / \mathrm{ml})$ increased the proportion of A2B5+ OPCs and O4+ immature oligodendrocytes in TSHI cultures $(P=0.009)$, as compared with parallel cultures grown without rEPO (Figure 2a). The effect of rEPO $(5 \mathrm{U} / \mathrm{ml})$ on $\mathrm{A} 2 \mathrm{~B} 5+$ or $\mathrm{O} 4+$ cells in sham cultures was less substantial, although a trend toward improved survival was noted. In both TSHI and sham cultures, treatment with EPOR-neutralizing antibodies caused a significant reduction in the proportion of $\mathrm{A} 2 \mathrm{~B} 5+/ \mathrm{O} 4+$ cells (both $P<$ 0.001 ), an effect not observed with nonspecific-isotype control antibodies.

To more specifically test the impact of EPO signaling on O4+ immature oligodendrocyte survival after injury, we used a pharmacologic model of cell death. Addition of 20 or 100 $\mu \mathrm{mol} / \mathrm{l}$ glutamate without $\mathrm{rEPO}$ for $2 \mathrm{~d}$ caused a dose-dependent decrease in $\mathrm{O} 4+$ cell survival of 29 and $57 \%(P<0.05)$ (Figure $2 \mathrm{~b}, \mathrm{c})$. With no glutamate present to induce additional EPOR expression, naive O4+ cells with basal levels of EPOR expression demonstrated minimal changes in survival following $\mathrm{rEPO}$ treatment $(0.1 \mathrm{or} 10 \mathrm{U} / \mathrm{ml})$. In the presence of an insult induced by low concentrations of glutamate $(20 \mu \mathrm{mol} / \mathrm{l})$, rEPO $(0.1$ or $10 \mathrm{U} / \mathrm{ml}$ ) improved $\mathrm{O} 4+$ cell survival back up to control levels. Following a challenge with a high concentration of glutamate $(100 \mu \mathrm{mol} / \mathrm{l})$ and $\mathrm{rEPO}$ treatment, survival improved in a dose-dependent manner, with $10 \mathrm{U} / \mathrm{ml} \mathrm{rEPO}(P=0.004) \mathrm{dem}$ onstrating more improvement than $0.1 \mathrm{U} / \mathrm{ml} \mathrm{rEPO}$. The effects of rEPO were blocked by EPOR-neutralizing antibodies but not nonspecific-isotype control antibodies. Specifically, survival decreased threefold after addition of EPOR-neutralizing antiEPOR antibodies to cultures incubated with $0.1 \mathrm{U} / \mathrm{ml} \mathrm{EPO} \mathrm{and}$ $20 \mu \mathrm{mol} / \mathrm{l}$ glutamate $(P=0.001)$. Similarly, heat-inactivated (hi) $\mathrm{rEPO}(10 \mathrm{U} / \mathrm{ml})$ also failed to improve survival in the presence of glutamate $(20 \mu \mathrm{mol} / \mathrm{l} ; P=0.009)$.

\section{EPO Signaling Does Not Alter Immature Oligodendrocyte Proliferation}

To determine whether enhanced proliferation contributed to the increase in the proportion of A2B5+ OPCs and O4+ immature oligodendrocytes observed in the presence of rEPO, 5-bromo-2'-deoxyuridine (BrdU) was added to $\mathrm{P} 2+3$ mixed cell cultures obtained from TSHI or sham controls. As expected, an increase in BrdU-positive cells was noted in 
untreated TSHI cultures compared with untreated sham cultures. Specifically, after TSHI injury, there was a $17 \%$ increase in the proportion of all BrdU+ cells. Of note, addition of $\mathrm{rEPO}$ $(5 \mathrm{U} / \mathrm{ml})$ did not alter the proportion of $\mathrm{A} 2 \mathrm{~B} 5+/ \mathrm{O} 4+$ cells double labeled with BrdU in either sham cultures or TSHI cultures. Furthermore, rEPO treatment did not alter the proportion of all BrdU+ cells in sham cultures, indicating that rEPO does not alter the proliferation of nonoligodendroglial neural cell types in mixed glial cultures.

\section{EPO Signaling Enhances Oligodendrocyte Maturation}

The hallmark of oligodendrocyte maturation is process formation. To assess the impact of rEPO signaling on process formation, we measured the footprint of processes made by individual $\mathrm{O} 4+$ immature oligodendrocytes (21). Addition of 1 and
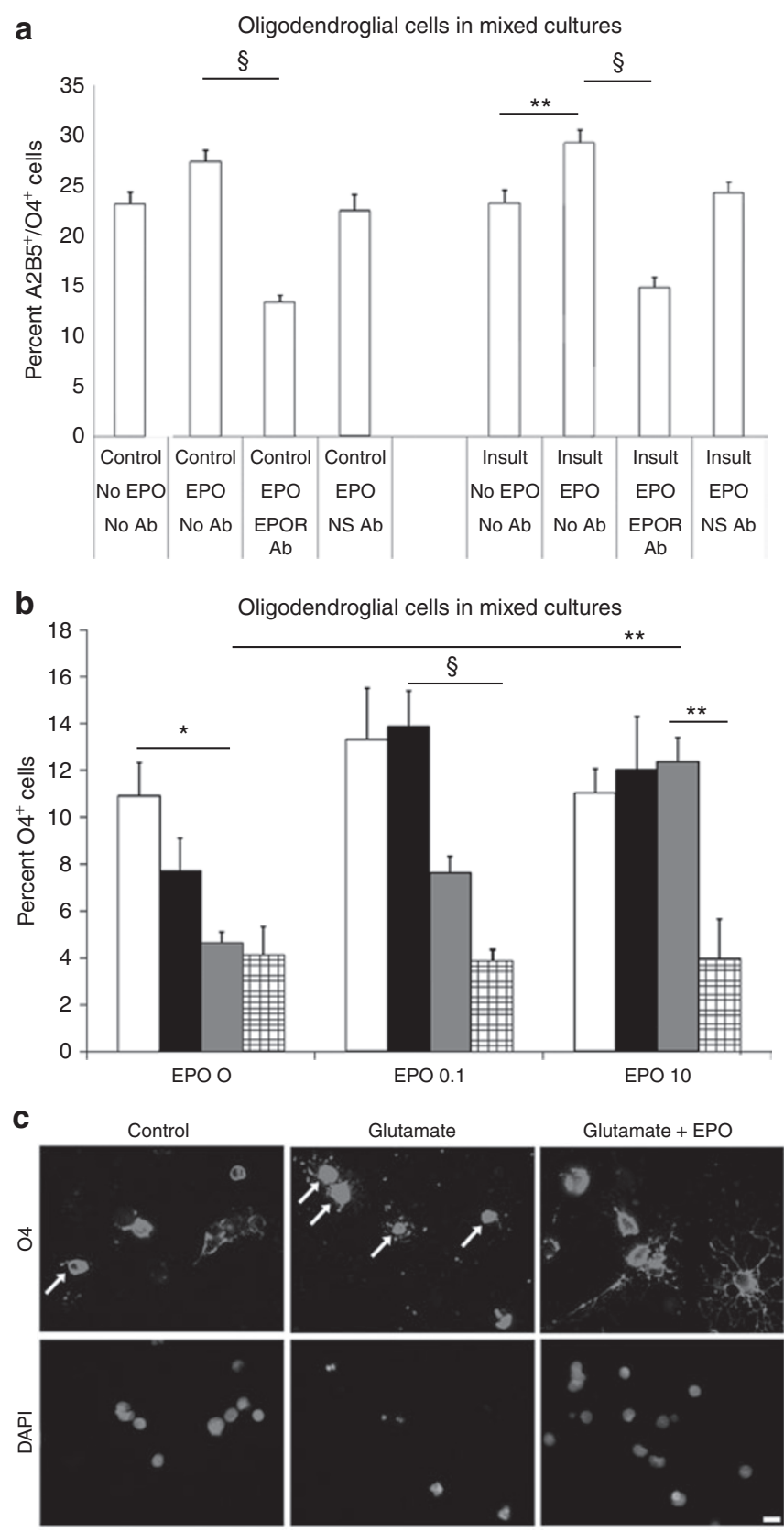

Glutamate + EPO

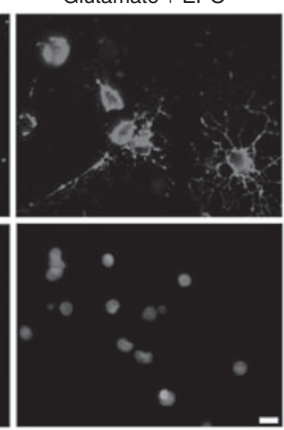

$5 \mathrm{U} / \mathrm{ml} \mathrm{rEPO}$ to cultures from shams caused a dose-dependent 9 and $41 \%$ increase, respectively, in the area occupied by $\mathrm{O} 4+$ processes as compared with cultures without $\mathrm{rEPO}$ (Figure 3a,b). Prenatal TSHI caused a marked decrease in the area occupied by $\mathrm{O} 4+$ cell processes $(P<0.001)$. Addition of 1 or $5 \mathrm{U} / \mathrm{ml} \mathrm{rEPO}$ to TSHI cultures returned the area of O4+ processes to levels comparable with shams with rEPO (both $P<0.001)$. In cultures from both shams and TSHI brains, rEPO's effect on process formation was abolished with either hi-rEPO or EPOR-neutralizing antibodies (all $P<0.001$ ), but not by nonspecific-isotype control antibodies. To specifically test whether the rEPO effect on $\mathrm{O} 4+$ process extension was due to glutamate-mediated toxicity, we examined $\mathrm{O} 4+$ process extension with varying doses of glutamate and rEPO in cultures from P5 naive control brains incubated for 2 DIV (Figure 4a,b). Addition of $0.1 \mathrm{U} / \mathrm{ml} \mathrm{rEPO}$ increased the area occupied by $\mathrm{O} 4+$ cell processes, compared with without $\mathrm{rEPO}$ $(P=0.011)$. When glutamate $(20 \mu \mathrm{mol} / \mathrm{l})$ was added to mimic excitotoxic $\mathrm{PBI}$, a significant loss in $\mathrm{O} 4+$ process area occurred $(P=0.002)$. The addition of 0.1 or $10 \mathrm{U} / \mathrm{ml} \mathrm{rEPO} \mathrm{to} \mathrm{cultures}$ with $20 \mu \mathrm{mol} / \mathrm{l}$ of glutamate restored $\mathrm{O} 4+$ cell process area to baseline (both $P<0.001)$. The effect of rEPO was blocked by EPOR-neutralizing antibodies $(P<0.001)$. In the presence of high dosages of glutamate $(100 \mu \mathrm{mol} / \mathrm{l}), \mathrm{rEPO}$ at $0.1 \mathrm{U} / \mathrm{ml}$ was insufficient to restore process formation, but $\mathrm{rEPO}$ at $10 \mathrm{U} / \mathrm{ml}$ was effective $(P=0.001)$, showing a dose-dependent response.

\section{EPO Enhances MBP Production After Injury}

Myelin is the functional outcome of oligodendroglial development, and exogenous rEPO enhances myelin production in vivo (6). To directly determine whether EPO signaling may contribute to MBP expression, cultures from P2 sham control or TSHI brains were incubated for 5 DIV. Addition of rEPO $(5 \mathrm{U} / \mathrm{ml})$ increased the MBP present in cultures from sham brains by $20 \%$ (Figure 5a,b). By contrast, in TSHI cultures, rEPO induced a $37 \%$ increase in MBP production $(P=0.012$; Figure $5 \mathrm{~b})$.

Figure 2. Erythropoietin supports survival of developing oligodendendroglial lineage cells. (a) Mixed glial cultures from P2 sham or transient systemic hypoxia-ischemia (TSHI) brains were incubated for $3 \mathrm{~d}$ in vitro and immunolabeled with $\mathrm{A} 2 \mathrm{~B} 5$ and $\mathrm{O} 4$ antibodies to identify OPCs and immature oligodendrocytes, respectively, and DAPI. Without recombinant erythropoietin (rEPO) treatment, no difference between TSHI- and sham-immunolabeled cell survival exists. rEPO treatment $(5 \mathrm{U} / \mathrm{ml})$ increases survival, and this effect is stronger following TSHI $(* * P=0.009)$. EPOR-neutralizing antibodies markedly decrease survival ( $\left({ }^{P} P<0.001\right)$, but nonspecific-isotype antibodies (NS Ab) do not. (b) To test the dose dependence of rEPO's support of O4+ cell survival, P5 mixed cells from naive brains were cultured with or without glutamate (white columns). Without rEPO, increasing doses of glutamate $(20$ and $100 \mu \mathrm{mol} / \mathrm{l}$, black and gray columns, respectively) causes a stepwise decrease in O4+ cell survival $\left({ }^{*} P=0.05\right)$. When cultures are challenged with glutamate, $r E P O$ treatment produces a dose-dependent increase in O4+ cell survival. With 100- $\mathrm{mmol} / \mathrm{I}$ glutamate, $r E P O 0.1 \mathrm{U} / \mathrm{ml}$ does not change survival, but $r E P O 10 \mathrm{U} / \mathrm{ml}$ was effective ( $\left.{ }^{*} P=0.004\right)$. In cultures with $\mathrm{rEPO} 0.1 \mathrm{U} / \mathrm{ml}$ and glutamate 20 $\mu \mathrm{mol} / \mathrm{l}$, EPOR-neutralizing antibodies (hatched columns) cause a marked decrease in survival ( $\left.{ }^{(} P=0.001\right)$. Similarly, heat-inactivated (hi)-rEPO $10 \mathrm{U} /$ $\mathrm{ml}$ added to cultures with glutamate $20 \mu \mathrm{mol} / \mathrm{l}$ did not improve survival $(* * P=0.009)$. (c) Photomicrographs of $\mathrm{O} 4+$ cells show fragmentation of $\mathrm{DAPI}+$ nuclei when dead or dying (arrows). Bar $=10 \mu \mathrm{m}$. 
a

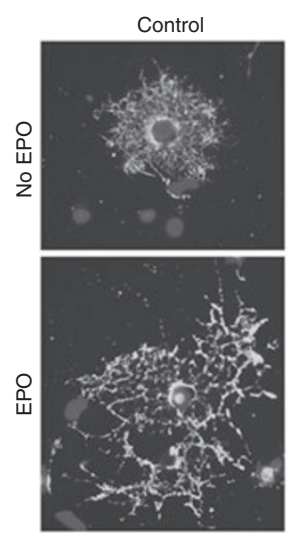

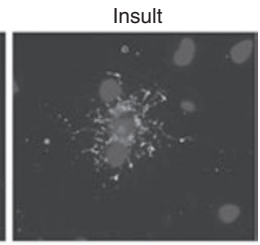
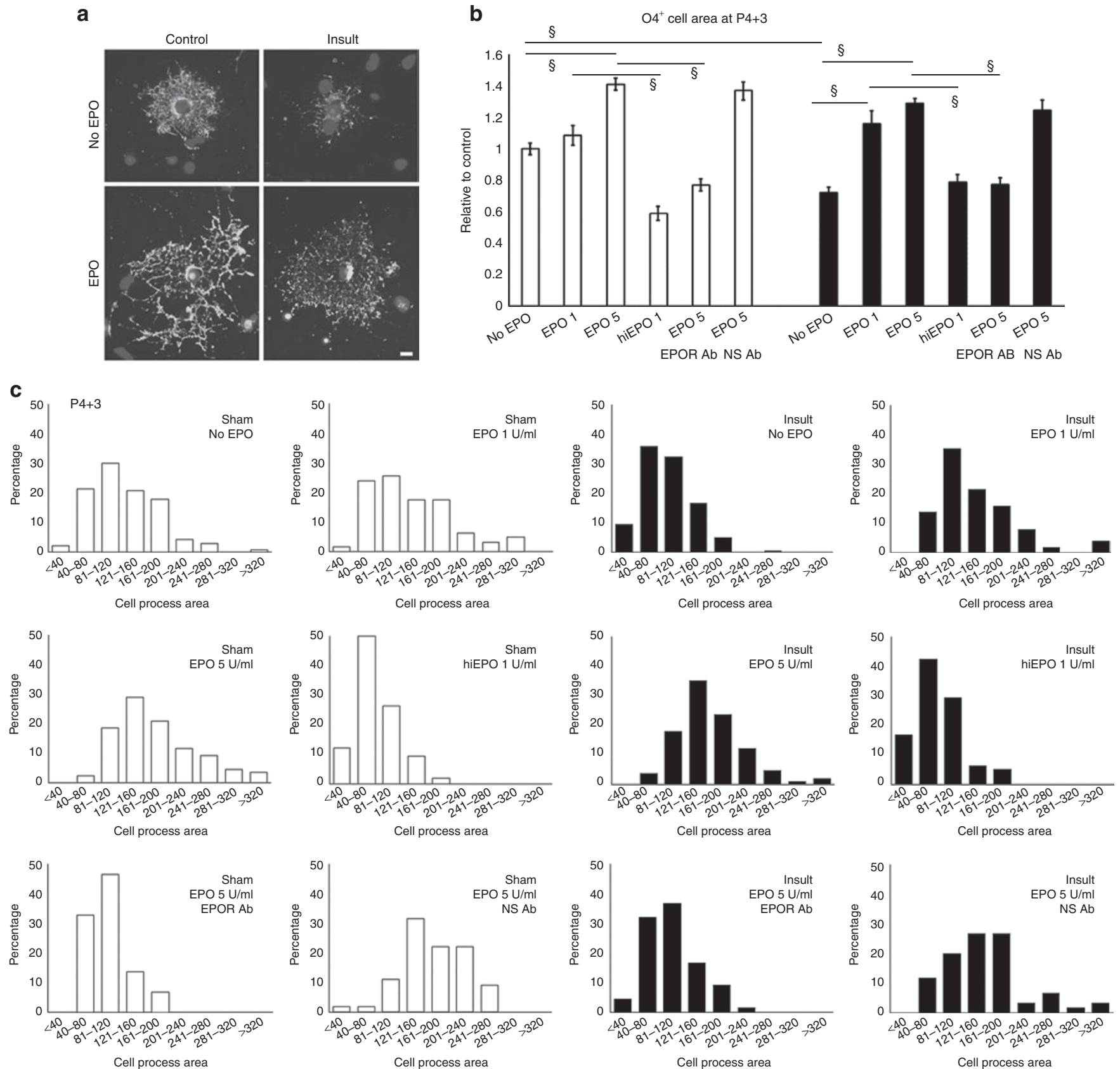

Figure 3. To assess erythropoietin (EPO)'s effect on $\mathrm{O} 4+$ immature oligodendrocyte process extension, cells from P4 sham (white columns) or transient systemic hypoxia-ischemia (TSHI) brains (black columns) were cultured $3 \mathrm{~d}$ in vitro. (a) Photomicrograph shows process extension diminishes after TSHI. Recombinant erythropoietin (rEPO) treatment enhances process extension in O4+ cells from both sham and TSHI brains. Bar $=10 \mu \mathrm{m}$. (b) O4+ cell area at $\mathrm{P} 4+3$. To quantify process extension, the area demarcated by $\mathrm{O} 4+$ footprint was measured on $\mathrm{O} 4+$ cells with healthy round DAPI+ nuclei. TSHI limits $\mathrm{O} 4+$ cell process extension $\left({ }^{5} P<0.001\right)$. $\mathrm{rEPO}$ treatment increases process extension for $\mathrm{O} 4+$ cells from both sham and TSHI brains in a dose-dependent manner ( $\left.{ }^{(} P<0.001\right)$. By contrast, hi-rEPO $1 \mathrm{U} / \mathrm{ml}$ is ineffective $\left({ }^{(} P<0.001\right)$. Similarly, EPOR-neutralizing antibodies, but not nonspecific-isotype antibodies (NS Ab), induce a marked decrease in $\mathrm{O} 4+$ cell process extension. (c) To show the distribution of the areas covered by O4+ cell processes, distributions of cell process areas at $\mathrm{P} 4+3$ are plotted as a percent of total O4+ cells. rEPO treatment causes a dose-dependent right-ward shift in distributions, and conversely, inhibition of rEPO signaling with either hi-rEPO or EPOR-neutralizing antibodies caused a leftward shift.

EPO Does Not Alter White Matter Klotho Levels Following TSHI In Vivo

Corroborating our prior in vivo data demonstrate postnatal rEPO treatment after prenatal TSHI attenuates oligodendrocyte loss and significantly restores MBP levels in adult animals (6), our data here indicate that rEPO enhances oligodendrocyte survival, differentiation, maturation, and MBP production following injury in the absence of a significant proliferative effect in vitro. Of note, Klotho, an antiaging protein, has recently been shown to have similar effects on oligodendrocyte lineage cells (22). To test whether the rEPO-induced increase in oligodendrocyte maturation and MBP expression is mediated by 

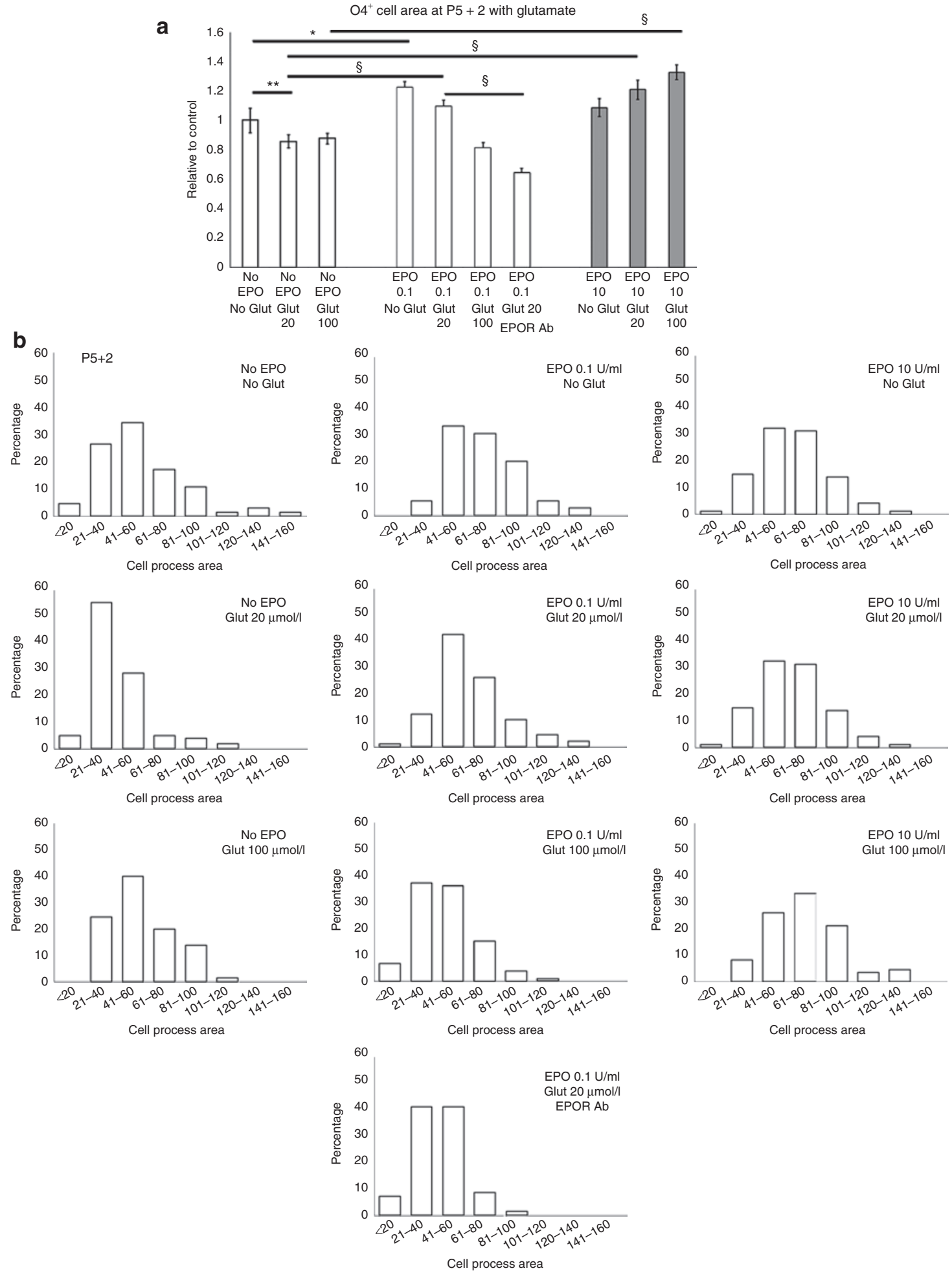

Figure 4. Loss of $\mathrm{O} 4+$ process extension induced by glutamate toxicity is restored by recombinant erythropoietin (rEPO) treatment at P5+2. (a) To more specifically test rEPO's effect on O4+ cell process extension, a glutamate assay was used to challenge P5 naive mixed cell cultures incubated for $2 \mathrm{~d}$ with $\mathrm{rEPO}(\mathrm{P} 5+2,0.1$, or $10 \mathrm{U} / \mathrm{ml}$; black and gray columns, respectively), glutamate $(20$ or $100 \mu \mathrm{mol} / \mathrm{l})$, and EPOR-neutralizing antibodies. Glutamate toxicity causes a decrease in area occupied by $\mathrm{O} 4+$ cell processes $\left({ }^{*} P=0.002 ;\right.$ no rEPO; white columns). $\mathrm{rEPO}$ treatment $(0.1 \mathrm{U} / \mathrm{ml})$ produces an increase in $\mathrm{O} 4+$ cell area $\left({ }^{*} P=0.011\right)$. With low-dose glutamate $(20 \mu \mathrm{mol} / \mathrm{l})$, both low- $(0.1 \mathrm{U} / \mathrm{ml})$ and high-dose $(10 \mathrm{U} / \mathrm{ml}) \mathrm{rEPO}$ effectively increase $\mathrm{O} 4+$ cell process area $\left({ }^{5} P<0.001\right)$. By contrast, with high-dose glutamate $(100 \mu \mathrm{mol} / \mathrm{l})$, only high-dose $\mathrm{rEPO}(10 \mathrm{U} / \mathrm{ml})$ is able to restore $\mathrm{O} 4+$ cell process area $\left({ }^{5} P=0.001\right)$, which confirms a dose-dependent effect of rEPO treatment on O4+ cell process extension. This rEPO effect was abolished by EPOR-neutralizing antibodies ( ${ }^{5} P$ $<0.001$ ). (b) To show the distribution of the areas covered by O4+ cell processes, distributions of O4+ cell process areas were plotted as a percent of the total $\mathrm{O} 4+$ cells. Glutamate shifts the distribution leftward, and this effect is mitigated by rEPO treatment in a dose-dependent manner. 
a

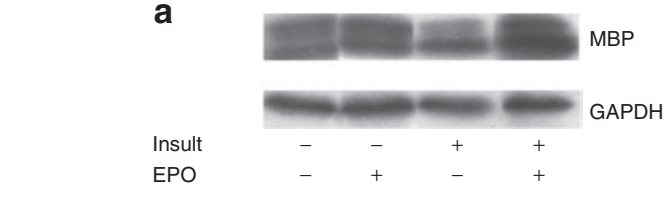

b

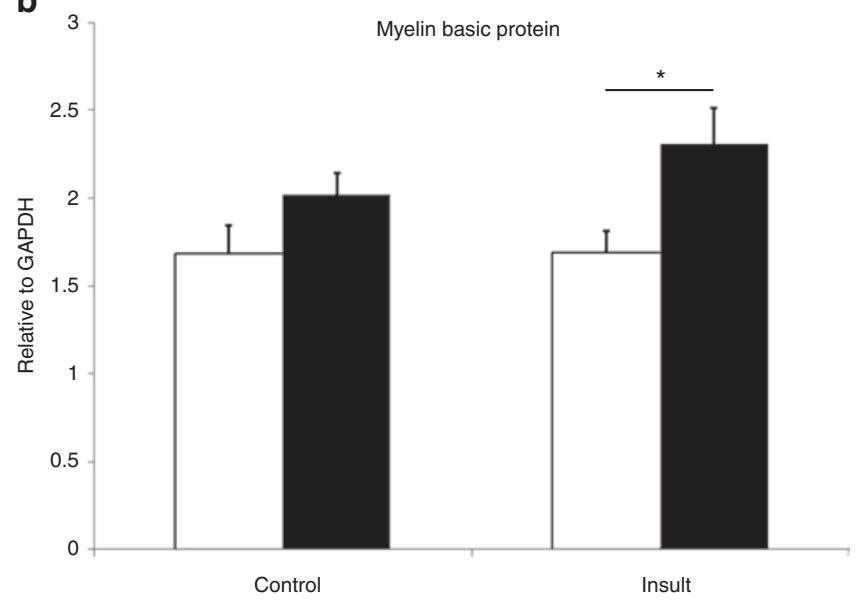

Figure 5. Recombinant erythropoietin (rEPO) treatment increases myelin basic protein expression in vitro after injury. $(\mathbf{a}, \mathbf{b})$ Mixed cell cultures from P2 sham or transient systemic hypoxia-ischemia (TSHI) brains were cultured for $7 \mathrm{~d}$ in vitro without (white columns) or with $\mathrm{rEPO}(5 \mathrm{U} / \mathrm{ml}$; black columns). TSHI reduces myelin basic protein (MBP) expression. rEPO treatment induces MBP expression in vitro, especially after prenatal TSHI $\left({ }^{*} P<0.05\right)$.

\section{a}

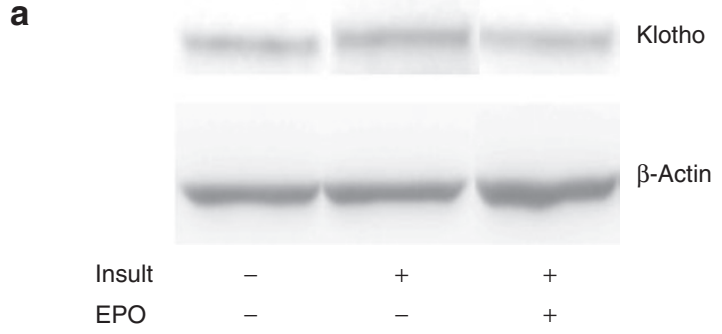

b

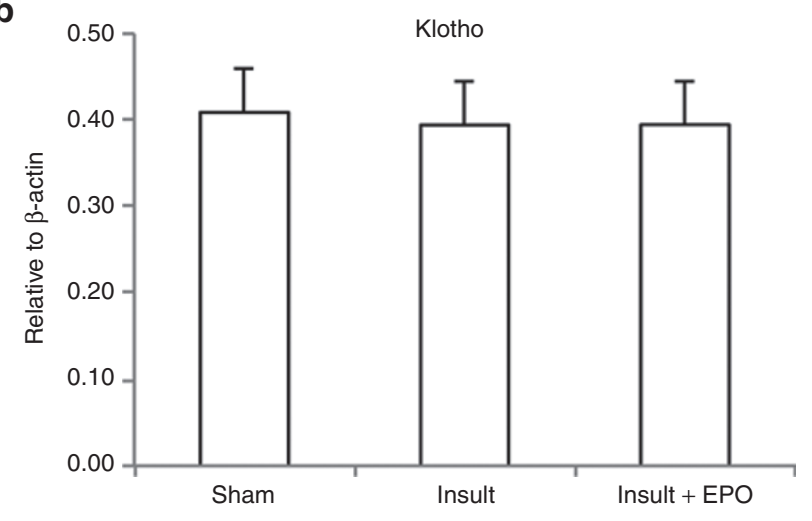

Figure 6. The antiaging protein, Klotho, is not affected by recombinant erythropoietin ( $\mathrm{rEPO}$ ) treatment in vivo. (a) White matter samples from P28 brain were incubated with anti-Klotho antibodies (130 kDa). (b) Klotho levels in rEPO-treated transient systemic hypoxia-ischemia (TSHI) white matter (Insult+EPO column) are not different from vehicle-treated TSHI (Insult column) or shams.
Klotho, we measured Klotho levels at P28 in sham and TSHI pups without and with postnatal rEPO treatment (Figure 6). As compared with sham animals $(n=5 ; 0.409 \pm 0.022)$, TSHI pups did not have significantly different levels of Klotho $(n=5$; $0.394 \pm 0.032)$. Neonatal rEPO-treated $(2,000 \mathrm{U} / \mathrm{kg} / \mathrm{d}$ intraperitoneally from P1 to P5) TSHI pups similarly had no change in Klotho levels at P28 $(n=5 ; 0.394 \pm 0.020)$.

\section{DISCUSSION}

EPO signaling controls multiple aspects of neuronal development $(8,9,16)$. Studies of functional outcome after rEPO treatment for neonatal WMI have shown efficacy associated with restored myelin levels (23), whereas delayed administration of exogenous rEPO after perinatal brain hypoxia-ischemia injury promotes myelin production and sustained functional improvement $(6,15)$. However, it is unclear whether the increase in myelin reflects a direct effect of EPO signaling on oligodendroglial lineage cell development or a secondary effect related to enhanced neuronal survival and development. While in vivo analyses allow a better assessment of persistent functional outcomes, in vitro analyses can better delineate specific regulatory steps in oligodendroglial development. Here, we show that rEPO promotes recovery of several stages in the development of the oligodendroglial lineage after PBI. Specifically, rEPO signaling regulates survival of OPCs and immature oligodendrocytes and process extension by $\mathrm{O} 4+$ immature oligodendrocytes in a dose-dependent manner. These results are interesting because in the recovery period following injury, OPCs tend to congregate around lesions but typically fail to progress through the stages of differentiation and maturation that lead to myelin production. Of note, we showed that $\mathrm{rEPO}$ increases $\mathrm{MBP}$ production in vitro. These effects of $\mathrm{rEPO}$ signaling on oligodendroglial lineage development in vitro were more potent following injury that mimics PBI than in sham controls. These findings are consistent with the hypothesis that injury to the developing brain increases EPOR expression without an adequate concomitant increase in ligand production $(6,24)$, creating excess vacant EPOR on neural cells. Our data indicate that this deficit can be reversed by rEPO treatment, resulting in enhanced oligodendroglial lineage recovery after injury.

Prior studies suggest that EPO is beneficial to oligodendrocyte development $(6,7,12,15)$. Oligodendroglial lineage cells express EPOR $(6,12,15,25,26)$, and EPOR expression increases after late gestation TSHI injury(6). Here, we confirmed that EPOR mRNA is elevated in oligodendrospheres after TSHI. In sham oligodendrospheres, rEPO induced a moderate $(74 \%)$ increase in PDGFaR mRNA levels as compared with oligodendrospheres with no added rEPO suggesting that during development, EPO signaling increases PDGF $\alpha$ R transcription and contributes to oligodendrogenesis. Furthermore, rEPO treatment significantly increased PDGF $\alpha$ R mRNA levels in TSHI cultures $(P=0.044)$, indicating that $\mathrm{rEPO}$ enhances the generation of new OPCs during development, and especially after injury to the developing brain. Increased EPOR expression after injury is supported by increased EPOR expression found in sheep after prenatal 


\section{Articles $\mid$ Jantzieet al.}

asphyxia (27) and on OPCs after lysolecithin injury in rats (28). Our data demonstrate for the first time that, similar to neurons, rEPO enhances the generation of new oligodendrocyte lineage cells from oligodendrospheres, and that this effect is enhanced following prenatal TSHI injury. These results are corroborated by prior studies in adult rats which showed more oligodendroglial lineage cells that were present following rEPO treatment of focal stroke $(29,30)$. Significantly, generation of new OPCs that will eventually mature and form myelin likely contributes to the sustained functional improvement resulting from rEPO treatment initiated in a delayed paradigm after a perinatal insult (6).

EPO promotes survival of A2B5+ OPCs and O4+ immature oligodendrocytes in a dose-dependent manner following TSHI and glutamate excitotoxicity. These results suggest that signaling from vacant EPOR triggers loss of oligodendrocyte lineage cells that can be reversed through rEPO treatment, and implicates this pathway in the regulation of the number of immature oligodendrocytes surviving during development. Pretreatment with $\mathrm{rEPO}$ is known to support mature oligodendrocyte survival in vitro in a dose-dependent manner following lipopolysaccharide and interferon- $\gamma$-induced injury (25). In addition, astrocyte-secreted EPO supports OPC and immature oligodendrocyte survival in vitro (26). Together with our results using EPOR-neutralizing antibodies, these data suggest endogenous EPO signaling contributes significantly to the generation and survival of developing oligodendroglial lineage cells, and supports our hypothesis that adequate EPO ligand contributes to neuronal and oligodendroglial survival after PBI. These data also suggest that EPO exerts a direct effect on target cells since exogenous $\mathrm{rEPO}$ rescues immature oligodendrocytes from glutamate-induced cell death in a dose-dependent manner.

We did not find a significant impact of rEPO signaling on OPC or immature oligodendrocyte proliferation. Combined with the survival data mentioned above, these data indicate that EPO increases the number of oligodendrocyte lineage cells primarily by enhancing survival and not by proliferation. Earlier in vivo data indicate that hypoxia-ischemia injury on $\mathrm{P} 7$ with delayed rEPO treatment increased the number of NG2+ cells, but their analyses did not distinguish the difference between survival and proliferation (15). Contrary to our findings that rEPO did not affect proliferation in mixed primary cultures immunolabeled with BrdU and A2B5/O4 antibodies, rEPO treatment increased BrdU+ proliferation in N20.1 cells, an immature oligodendrocyte cell line (30). It is not unexpected that a cell line responded differently than primary cultures given that the EPOR expression is highly dynamic. Our observations that $\mathrm{rEPO}$ signaling did not increase the number of BrdU+ cells in our model, and does not appear to drive proliferation, is reassuring. In the thousands of infants treated with rEPO for anemia of prematurity and in the clinical trials that have been performed thus far in neonates with brain injury, no evidence of tumor growth has been reported (5,31-33).

EPO increased O4+ cell process extension, a sign of oligodendrocyte morphogenesis and maturation. Following various brain injuries, including in models of periventricular leukomalacia and hypoxic-ischemic injury in the developing brain, OPCs and O4+ immature oligodendrocytes often fail to survive and do not become mature myelin-producing mature oligodendrocytes (20). Here, we used glutamate excitotoxicity and EPOR-neutralizing antibodies to demonstrate that rEPO treatment induced process formation by $\mathrm{O} 4+$ cells in a dosedependent manner, which may address the hurdle to restoration of myelination. Our detailed analyses extend previous findings that showed improved process formation with $\mathrm{rEPO}$ treatment $(7,28)$. Multiple molecules regulate process formation by $\mathrm{O} 4+$ cells, including secreted extracellular matrix proteins, regulators of cell cycle exit, and mediators of oxidative stress, and therefore are candidate mediators of the effect of rEPO on immature oligodendrocyte process extension. Previously, we documented that postnatal $\mathrm{rEPO}$ treatment after prenatal TSHI-attenuated oligodendrocyte and GABAergic neuronal cell loss, and significantly restored MBP levels in injured animals compared with shams (6). Recently, the antiaging protein, Klotho, has been shown to enhance oligodendrocyte maturation and myelination in the rodent central nervous system (22). The effects of Klotho and EPO on oligodendroglial lineage cell survival and maturation are similar, and Klotho signals through Akt (22), as does EPO (9). Therefore, we investigated whether Klotho levels were altered in rEPO-treated TSHI pups in vivo. On P28, EPO-treated TSHI pups did not have significantly different white matter Klotho levels compared with sham or vehicle-treated TSHI pups. These results suggest that despite similarities, different mechanisms probably mediate Klotho and EPO regulation of oligodendrocyte maturation during development and after injury. It is reasonable that multiple pathways are affected by $\mathrm{rEPO}$ treatment given the diverse mechanisms involved in EPO signaling. For example, during early precursor cell specification, bone morphogenic protein 4 (BMP4) drives precursors toward the astroglial lineage instead of the oligodendroglial lineage (34). In a model of prenatal (E19) permanent systemic hypoxia-ischemia, high BMP4 levels were associated with an increased oxidative stress and inhibition of OPC differentiation, and fewer and shorter $\mathrm{GalC}+$ mature oligodendrocyte processes (35). EPO signaling mitigates oxidative stress $(25,36)$, and therefore EPO signaling may also serve limit free radical-induced BMP4 levels. These candidate molecules and pathways represent examples that may be integral to EPO's effects on myelination and are subjected to future investigation.

The failure of newly generated OPCs and $\mathrm{O} 4+$ immature oligodendrocytes to mature has been a major obstacle in strategies to improve myelination after central nervous system injury. Treatment with neuroprotective rEPO dosages appears to promote process formation by immature oligodendrocytes, and may circumvent this blockade. We found that in cultures from both in vitro and in vivo models of PBI, rEPO demonstrated a dose-dependent effect on the area of process formation by $\mathrm{O} 4+$ immature oligodendrocytes. Furthermore, the enhanced $\mathrm{O} 4+$ process formation with rEPO treatment coincided with increased MBP expression, especially in neural cells that suffered a prenatal TSHI insult. Consistent with our 
findings that rEPO treatment increased MBP expression, others have shown that addition of rEPO improves MBP expression after injury $(11-15,23,28,30)$. Also, in extended cultures of immature rat oligodendrocytes, addition of rEPO increased the process formation and the number of MBP-positive cells (7). Several of the signaling pathways mentioned above are involved in myelination as well as process extension, and probably also mediate the enhancement of MPB expression induced by rEPO treatment.

EPO has multiple actions as a neuroprotective agent including enhancing neurogenesis after injury $(16,17)$, neuronal survival after injury (14), decreasing toxic-free radical oxidants $(25,36)$, decreasing proinflammatory cytokines (37), and microglial-mediated recruitment and toxicity (13). One limitation of this study is that we used a model of hypoxia-ischemia without a component of inflammation. Chorioamnionitis is common in infants born very preterm (1). The impact of inflammation of the efficacy of EPO treatment needs to be clarified, and such studies are currently ongoing. rEPO treatment up to a week after injury may be beneficial because injury-induced increased expression of EPOR persists for days $(6,24)$. A significant component of neuroprotection delivered by rEPO administration after injury probably reflects the promotion of new waves of neural cells to replace those lost by injury. Of note, here, we have shown that rEPO treatment after injury enhances the genesis of OPCs, survival, and maturation of immature oligodendrocytes and MBP expression. Early clinical trials in neonates with PBI have shown that neuroprotective rEPO dosages are safe and do not increase adverse events in the short or long term (5,31-33). As the indications for rEPO treatment of injury to the developing brain expand and are refined, it is critical to clearly delineate the effects of rEPO treatment on the injured developing brain.

\section{METHODS}

\section{Prenatal Transient Systemic Hypoxia-Ischemia}

All procedures were performed in accordance with the National Institutes of Health (Bethesda, MD) Guide for the Care and Use of Laboratory Animals and with the approval of Institutional Animal Care and Use Committee at Case Western Reserve University. An established model of prenatal TSHI was used (3). Briefly, on E18, Sprague-Dawley rats were anesthetized with isoflurane, and uterine arteries were clamped for $60 \mathrm{~min}$. Sham control dams underwent anesthesia and laparotomy for $60 \mathrm{~min}$ without uterine artery occlusion. Pups were born at term. Fetal loss was $23 \%$ after TSHI and 0 in shams. A subset of pups were treated with intraperitoneal $\mathrm{rEPO}$ $(2,000 \mathrm{U} / \mathrm{kg} / \mathrm{d})$ or vehicle (saline) from P1 to P5 (6).

\section{Oligodendrospheres}

Mixed neural single-cell suspensions were made $(3,6)$ using two E19 control or two TSHI brains for each experiment. Each experiment was performed three times using six brains total per condition. Cell suspensions were placed in flasks containing oligodendrocyte supportive media (OSM) (F12+N2;10 ng/ml PDGF) with $1 \%$ fetal calf serum and 10-ng/ml epidermal growth factor, and grown for 2 DIV (E19+2) (18). On 3 DIV, oligodendrospheres from each culture were separated into two flasks with serum-free (SF) OSM, and $\mathrm{rEPO}(5 \mathrm{U} / \mathrm{ml}$; R\&D Systems, Minneapolis, MN) was added to one flask. After $6 \mathrm{DIV}$, cells were collected for quantitative PCR for EPOR, PDGFaR, and ribosomal 18s (endogenous standard).
Primers were EPOR-forward: GACCCCAGCTCTAAGCTCCT, EPOR-reverse: AGCCCCCTGAGCTGTAATCT, PDGFaR-forward: CTGGTGCCTGCCTCCTAC, PDGFaR-reverse: AACTCGCTGGTC TTGAACG, 18s-forward: TCCCTAGTGATCCCCGAGAAGT, and 18s-reverse: CCCTTAATGGCAGTGATAGCGA (Integrated DNA Technologies, Coralville, IA). cDNA was added to Sybr green (Kapa Biosystems, Woburn, MA) and run on a Realplex2 Mastercycler (Eppendorf, Hauppauge, NY). Cycle thresholds from oligodendrospheres were compared with cycle thresholds values from P2 sham frontal lobe, with PDGFaR or EPOR levels normalized to 18s. Samples were run in triplicate, and three independent experiments were performed. For immunocytochemistry, on E19+5, a random subset of oligodendrospheres from each flask was plated overnight on polyL-lysine-coated coverslips to allow immunolabeling with antibodies to A2B5+ and $\mathrm{O} 4+$ to label OPCs and immature oligodendrocytes, respectively. Primary antibodies were visualized with antimouse fluorescein-conjugated secondary antibodies. Cells were double-labeled with 4',6-diamidino-2-phenylindole (DAPI) to identify cell nuclei. Representative oligodendrospheres were photographed on a Leica microscope.

\section{In Vitro Survival Assays}

Immature oligodendrocytes express glutamate receptors $(4,38,39)$, glutamate toxicity induces neural cell death in a dose-dependent manner $(9,40)$, and glutamate mediates PBI $(38,39)$. To directly determine whether rEPO can rescue injured developing neural cells in vitro, we performed a glutamate cell survival assay (40). Mixed single-cell suspensions from P5 naive brains were plated at 50,000 cells/poly-L-lysinecoated coverslip and cultured overnight in OSM+1\% fetal calf serum. On P5+1, cells were transferred to SF-OSM and rEPO, heat-inactivated rEPO, glutamate, EPOR-neutralizing antibodies (M-20; Santa Cruz, Dallas, TX), or nonspecific-isotype control antibodies were added. On $\mathrm{P} 5+2$, cells were immunolabeled with $\mathrm{A} 2 \mathrm{~B} 5$ and $\mathrm{O} 4$ antibodies, and mounted with DAPI. Live cells had round, well-formed DAPI+ nuclei, whereas dead cells had no DAPI labeling, and dying cells had pyknotic DAPI+ nuclei. Total number of live cells and proportions of A2B5+ or $\mathrm{O} 4+$ cells were counted at $\times 20$ on $>5$ random fields/coverslip from four to six coverslips/condition from at least two separate experiments. The proportion of live immature oligodendroglial cells was calculated.

\section{In Vitro Proliferation Assays}

For proliferation assays, mixed glial single-cell suspensions from P2 sham or TSHI brains were plated at 50,000 cells/coverslip, incubated overnight in $\mathrm{OSM}+1 \%$ fetal calf serum, and on $\mathrm{P} 2+1$, cells were transferred to SF-OSM. BrdU $(50 \mu \mathrm{mol} / \mathrm{l})$ was added for $16 \mathrm{~h}$ before immunolabeling on $\mathrm{P} 2+3$ with $\mathrm{A} 2 \mathrm{~B} 5, \mathrm{O} 4$, and anti-BrdU antibodies as previously described (3). The number of live DAPI+ total cells, A2B5/O4+ cells, BrdU+ cells, and A2B5/O4+/BrdU+ cells were counted on $>5$ random fields at $\times 20$ on three to four coverslips/condition from three separate experiments. The proportion of immature oligodendroglial cells (A2B5+/O4+cells/total cells), proliferating total cells (BrdU+ cells/total cells), and proliferating immature oligodendroglial cells (A2B4+/O4+/BrdU+ cells per A2B5+/O4+ cells) were calculated.

\section{Oligodendrocyte Process Formation}

Mixed glial single-cell suspensions were prepared as above from $\mathrm{P} 4$ sham or TSHI pups, or P5 naive control pups for glutamate doseresponse studies. After an overnight culture on coverslips in OSM $+1 \%$ FBS, cells were transferred to SF-OSM. Cells were immunolabeled on P7 equivalent with O4+ antibodies and DAPI. Randomly selected individual $\mathrm{O} 4+$ cells with round healthy appearing DAPI+ nuclei were photographed on a Leica microscope. The footprint made by each cell's processes was measured using Photoshop CS4 Extended (Adobe, San Jose, CA) (21). At least 50 cells/condition from more than three separate coverslips (mean 108; range: 51-198) were measured from two or more separate experiments.

\section{Western Blot for MBP and Klotho}

Mixed glial single-cell suspensions from P2 sham control or TSHI brains were plated on poly-L-lysine-coated 60 -mm culture dishes with 
6 million cells/dish. After an overnight incubation in OSM $+1 \%$ fetal calf serum, cultures were transferred to SF-OSM with $\mathrm{rEPO}(5 \mathrm{U} / \mathrm{ml})$ replenished every $48 \mathrm{~h}$. After $7 \mathrm{DIV}$, cells were lysed and centrifuged. After electrophoresis on 4-12\% Tris-glycine gels, proteins were transferred to polyvinylidene difluoride membranes, incubated with antiMBP (Covance SMI-99 at 1:1,000), or anti-glyceraldehyde-3-phosphate dehydrogenase antibodies (sc-697; 1:10,000; Santa Cruz), and probed with species-appropriate horseradish peroxidase-conjugated secondary antibodies. Relative optical density for each band was measured using Image J (National Institutes of Health). Three separate experiments were performed. Western blots for Klotho were performed on soluble proteins extracted from white matter microdissected from five P28 brains per group as above. Membranes were then probed with anti-Klotho (1:500; R\&D Systems) or anti-actin (1:5,000; Sigma, St. Louis, MO) antibodies.

\section{Quantification and Statistics}

Observers were blinded to insult and treatment status. Each experiment used animals from at least two different litters. Data are presented as mean with SEM. Differences were compared with two-way ANOVA with Bonferroni's correction for multiple pairwise comparisons using SPSS19 (IBM, St. Louis, MO), with $P<0.05$ considered significant.

\section{ACKNOWLEDGMENTS}

We appreciate the excellent technical assistance of Qing Li, Elizabeth Shick, and especially Mark Eden.

\section{STATEMENT OF FINANCIAL SUPPORT}

This study was supported by the National Institute of Neurological and Stroke (Bethesda, MD) grant R01-NS060765.

Disclosure: There is no conflict of interest to disclose.

\section{REFERENCES}

1. Volpe JJ. Brain injury in premature infants: a complex amalgam of destructive and developmental disturbances. Lancet Neurol 2009;8:110-24.

2. Kessaris N, Fogarty M, Iannarelli P, Grist M, Wegner M, Richardson WD. Competing waves of oligodendrocytes in the forebrain and postnatal elimination of an embryonic lineage. Nat Neurosci 2006;9:173-9.

3. Robinson S, Petelenz K, Li Q, et al. Developmental changes induced by graded prenatal systemic hypoxic-ischemic insults in rats. Neurobiol Dis 2005;18:568-81.

4. Jantzie LL, Talos DM, Selip DB, et al. Developmental regulation of group I metabotropic glutamate receptors in the premature brain and their protective role in a rodent model of periventricular leukomalacia. Neuron Glia Biol 2010;6:277-88.

5. McPherson RJ, Juul SE. Erythropoietin for infants with hypoxic-ischemic encephalopathy. Curr Opin Pediatr 2010;22:139-45.

6. Mazur M, Miller RH, Robinson S. Postnatal erythropoietin treatment mitigates neural cell loss after systemic prenatal hypoxic-ischemic injury. J Neurosurg Pediatr 2010;6:206-21.

7. Sugawa M, Sakurai Y, Ishikawa-Ieda Y, Suzuki H, Asou H. Effects of erythropoietin on glial cell development; oligodendrocyte maturation and astrocyte proliferation. Neurosci Res 2002;44:391-403.

8. Knabe W, Knerlich F, Washausen S, et al. Expression patterns of erythropoietin and its receptor in the developing midbrain. Anat Embryol 2004;207:503-12.

9. Digicaylioglu M, Garden G, Timberlake S, Fletcher L, Lipton SA. Acute neuroprotective synergy of erythropoietin and insulin-like growth factor I. Proc Natl Acad Sci USA 2004;101:9855-60.

10. Ruscher K, Freyer D, Karsch M, et al. Erythropoietin is a paracrine mediator of ischemic tolerance in the brain: evidence from an in vitro model. J Neurosci 2002;22:10291-301.

11. Fan X, Heijnen CJ, van der KOOIJ MA, Groenendaal F, van Bel F. Beneficial effect of erythropoietin on sensorimotor function and white matter after hypoxia-ischemia in neonatal mice. Pediatr Res 2011;69:56-61.

12. Kako E, Kaneko N, Aoyama M, et al. Subventricular zone-derived oligodendrogenesis in injured neonatal white matter in mice enhanced by a nonerythropoietic erythropoietin derivative. Stem Cells 2012;30:2234-47.
13. Liu W, Shen Y, Plane JM, Pleasure DE, Deng W. Neuroprotective potential of erythropoietin and its derivative carbamylated erythropoietin in periventricular leukomalacia. Exp Neurol 2011;230:227-39.

14. Yamada M, Burke C, Colditz P, Johnson DW, Gobe GC. Erythropoietin protects against apoptosis and increases expression of non-neuronal cell markers in the hypoxia-injured developing brain. J Pathol 2011;224:101-9.

15. Iwai M, Stetler RA, Xing J, et al. Enhanced oligodendrogenesis and recovery of neurological function by erythropoietin after neonatal hypoxic/ischemic brain injury. Stroke 2010;41:1032-7.

16. Shingo T, Sorokan ST, Shimazaki T, Weiss S. Erythropoietin regulates the in vitro and in vivo production of neuronal progenitors by mammalian forebrain neural stem cells. J Neurosci 2001;21:9733-43.

17. Wang L, Zhang Z, Wang Y, Zhang R, Chopp M. Treatment of stroke with erythropoietin enhances neurogenesis and angiogenesis and improves neurological function in rats. Stroke 2004;35:1732-7.

18. Pedraza CE, Monk R, Lei J, Hao Q, Macklin WB. Production, characterization, and efficient transfection of highly pure oligodendrocyte precursor cultures from mouse embryonic neural progenitors. Glia 2008;56:133952.

19. Haynes RL, Folkerth RD, Keefe RJ, et al. Nitrosative and oxidative injury to premyelinating oligodendrocytes in periventricular leukomalacia. J Neuropathol Exp Neurol 2003;62:441-50.

20. Segovia KN, McClure M, Moravec M, et al. Arrested oligodendrocyte lineage maturation in chronic perinatal white matter injury. Ann Neurol 2008;63:520-30.

21. Weng Q, Chen Y, Wang H, et al. Dual-mode modulation of Smad signaling by Smad-interacting protein Sip1 is required for myelination in the central nervous system. Neuron 2012;73:713-28.

22. Chen CD, Sloane JA, Li H, et al. The antiaging protein Klotho enhances oligodendrocyte maturation and myelination of the CNS. J Neurosci 2013;33:1927-39.

23. Li L, Jiang Q, Ding G, et al. MRI identification of white matter reorganization enhanced by erythropoietin treatment in a rat model of focal ischemia. Stroke 2009;40:936-41.

24. Schober ME, Block B, Beachy JC, Statler KD, Giza CC, Lane RH. Early and sustained increase in the expression of hippocampal IGF-1, but not EPO, in a developmental rodent model of traumatic brain injury. J Neurotrauma 2010;27:2011-20.

25. Genc K, Genc S, Baskin H, Semin I. Erythropoietin decreases cytotoxicity and nitric oxide formation induced by inflammatory stimuli in rat oligodendrocytes. Physiol Res 2006;55:33-8.

26. Kato S, Aoyama M, Kakita H, et al. Endogenous erythropoietin from astrocyte protects the oligodendrocyte precursor cell against hypoxic and reoxygenation injury. J Neurosci Res 2011;89:1566-74.

27. Castillo-Meléndez M, Yan E, Walker DW. Expression of erythropoietin and its receptor in the brain of late-gestation fetal sheep, and responses to asphyxia caused by umbilical cord occlusion. Dev Neurosci 2005;27:220-7.

28. Cho YK, Kim G, Park S, et al. Erythropoietin promotes oligodendrogenesis and myelin repair following lysolecithin-induced injury in spinal cord slice culture. Biochem Biophys Res Commun 2012;417:753-9.

29. Kim YJ, Jung YW. Systemic injection of recombinant human erythropoietin after focal cerebral ischemia enhances oligodendroglial and endothelial progenitor cells in rat brain. Anat Cell Biol 2010;43:140-9.

30. Zhang L, Chopp M, Zhang RL, et al. Erythropoietin amplifies strokeinduced oligodendrogenesis in the rat. PLoS ONE 2010;5:e11016.

31. Juul SE, McPherson RJ, Bauer LA, Ledbetter KJ, Gleason CA, Mayock DE. A phase I/II trial of high-dose erythropoietin in extremely low birth weight infants: pharmacokinetics and safety. Pediatrics 2008;122:383-91.

32. McAdams RM, McPherson RJ, Mayock DE, Juul SE. Outcomes of extremely low birth weight infants given early high-dose erythropoietin. J Perinatol 2013;33:226-30.

33. Wu YW, Bauer LA, Ballard RA, et al. Erythropoietin for neuroprotection in neonatal encephalopathy: safety and pharmacokinetics. Pediatrics 2012;130:683-91.

34. Miller RH, Dinsio K, Wang R, Geertman R, Maier CE, Hall AK. Patterning of spinal cord oligodendrocyte development by dorsally derived BMP4. J Neurosci Res 2004;76:9-19. 
35. Reid MV, Murray KA, Marsh ED, Golden JA, Simmons RA, Grinspan JB. Delayed myelination in an intrauterine growth retardation model is mediated by oxidative stress upregulating bone morphogenetic protein 4 . J Neuropathol Exp Neurol 2012;71:640-53.

36. Sifringer M, Brait D, Weichelt U, et al. Erythropoietin attenuates hyperoxiainduced oxidative stress in the developing rat brain. Brain Behav Immun 2010;24:792-9.

37. Sun Y, Calvert JW, Zhang JH. Neonatal hypoxia/ischemia is associated with decreased inflammatory mediators after erythropoietin administration. Stroke 2005;36:1672-8.
38. Manning SM, Talos DM, Zhou C, et al. NMDA receptor blockade with memantine attenuates white matter injury in a rat model of periventricular leukomalacia. J Neurosci 2008;28: $6670-8$.

39. Salter MG, Fern R. NMDA receptors are expressed in developing oligodendrocyte processes and mediate injury. Nature 2005;438: $1167-71$.

40. Oka A, Belliveau MJ, Rosenberg PA, Volpe JJ. Vulnerability of oligodendroglia to glutamate: pharmacology, mechanisms, and prevention. J Neurosci 1993;13:1441-53. 